\title{
Mitigating RIN-Penalty To Enhance The Transmission Performance In Distributed Raman Amplification System
}

\author{
Mingming Tan, Md Asif Iqbal, Sergei K. Turitsyn, and Paul Harper \\ 1 Aston Institute of Photonic Technologies, Aston University, Birmingham, B4 7ET, UK \\ m.tan1@aston.ac.uk
}

\begin{abstract}
The paper reviews three different technologies to mitigate the relative intensity noise (RIN) penalty in distributed Raman amplification system. We demonstrate the performance improvement in long-haul repeatered transmission systems, thanks to the signal RIN reduction.
\end{abstract}

Keywords - coherent communications; Raman amplification;

\section{INTRODUCTION}

It is well known that distributed Raman amplification (DRA) extends the maximum reach in long-haul transmission system, compared with discrete amplification, e.g. EDFA [1]. This is because the signal gain is "pushed" towards the middle of the transmission span and therefore signal gain is distributed uniformly over the fibre span, which leads to the reduction of amplified spontaneous emission (ASE) noise [2]. Currently, DRA usually relies on back (BW) propagated pumping which means the signal and the pump travels in opposite directions [3]. In order to achieve superior noise performance, BW pumping is far from enough. It is crucial to use forward (FW) propagated pumping with the signal traveling in the same direction as the pump light, which forms bidirectional Raman pumping. However, the signal can be easily affected by the relative intensity noise (RIN) of the Raman pumps [4]. In particular, in FW-pumped scenario, the signal and the pump light are propagated over the fibre at the same direction, and the noise from the pump is superimposed on the signal noise [5]. Consequently, the signal performance would be degraded severely in long-haul transmission systems due to RIN transfer. In [6], we showed that the maximum transmission distance was degraded from $7082 \mathrm{~km}$ (BW-pumping only) to only $1666 \mathrm{~km}$ using symmetric bidirectional pumping.

In this paper, different RIN mitigation techniques in DRA system are reviewed, including second order, first order, and dual order FW-pumping. We present long-haul transmission performance can be effectively improved, thanks to the RIN mitigation and ASE noise reduction.

\section{RIN MITIGATION AND LONG-HAUL TRANSMISSION PERFORMANCE IMPROVEMENT}

Pump/signal RIN suppression and its related penalty has been investigated in [7-9], but they have all focused on the application in unrepeatered transmission. The RIN penalty could be almost negligible over single span [10], but it would

Identify applicable sponsor/s here. If no sponsors, delete this text box (sponsors). be accumulated and become dramatic in long-haul repeatered transmission [6].

\section{A. FW-propagated second order pumping}

Schematic diagrams and pump powers for the Raman configurations are shown in Fig. 1(a). For all configurations, the Raman gain was set to counterbalance $\sim 16.5 \mathrm{~dB}$ attenuation of the $83 \mathrm{~km}$ SSMF fiber. At the output of the fibre span, the FBG (fibre Bragg grating) used was centered at $1455 \mathrm{~nm}$ with $\sim 0.5 \mathrm{~nm} 3 \mathrm{~dB}$ bandwidth and $95 \%$ reflectivity. Thanks to the BW-pumping at $1366 \mathrm{~nm}$, first order random distributed feedback (DFB) laser at $1455 \mathrm{~nm}$ was generated by the resonant mode reaching the lasing threshold in a distributed cavity formed by a distributed feedback (Rayleigh scattering) and an FBG [11-13]. Three pump power combinations (A1, A2, and A3) were used in this configuration, as forward pumping at $1366 \mathrm{~nm}$ could amplify the signal near the input of the fiber by amplifying the forward-propagated random DFB lasing.
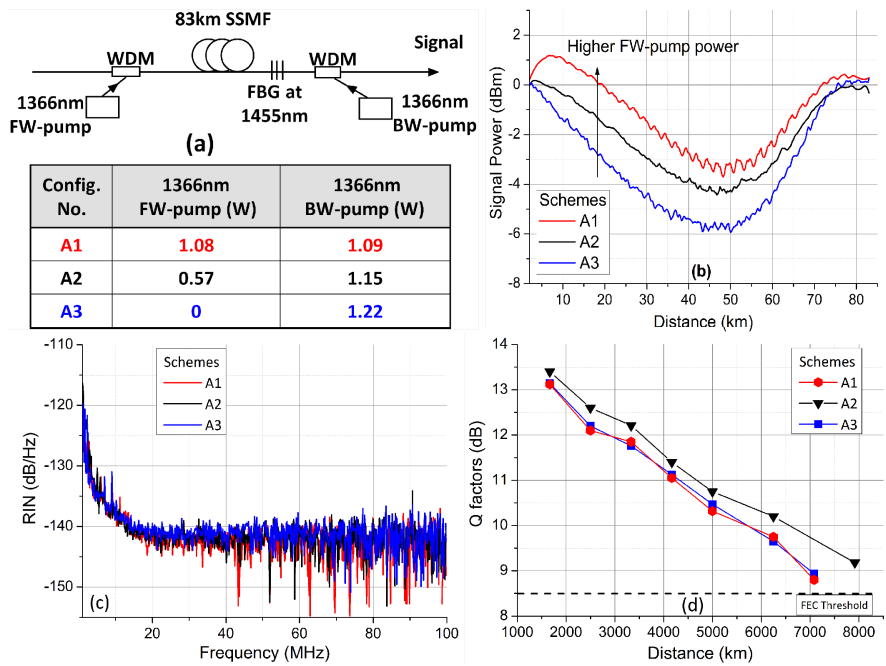

Figure 1. (a). Schematic of Raman fibre laser based amplifier using second order FW pumping. (b). Signal power profiles along the span using different FW pump power. (c). RIN of the signal using different FW pump power. (d). Q factors versus transmission distances using all the schemes.

As shown in Fig. 1(b), signal power profiles along the fibre span were measured at $1545.32 \mathrm{~nm}$ using a modified optical time-domain reflectometer setup. Compared with A3 (BWpumping only), the signal Raman gain can be clearly seen near the input section of the span and the ASE noise was reduced, thanks to the use of FW-pumping at $1366 \mathrm{~nm}$. Moreover, as 
shown in Fig. 1(c), the signal RIN remained the same for all the pumping schemes, which indicates the signal no longer suffered from the RIN-penalty. Fig. 1(d) shows the long-haul transmission of this Raman scheme using 10*100G DP-QPSK WDM transmitter and coherent receiver. The detailed setup can be found in [12]. The maximum reach using FW-pumping at $1366 \mathrm{~nm}$ was up to $7915 \mathrm{~km}$, better or similar to BW-pumping only scheme $(7082 \mathrm{~km})$. This reach was significantly improved, compared with $1666 \mathrm{~km}$, in which the signal suffered from the RIN-penalty as presented in [6]. Further investigations on the impact of signal RIN with different FBG reflectivity near the input end can be found in $[13,14]$.

\section{B. FW-propagated first order pumping}

The main drawback of scheme A was that the Raman gain efficiency near the input section was very low due to the use of second order pump in the forward direction with no seed of first order pump. Using first order FW pump instead of second order FW pump can improve the Raman gain efficiency and require much lower pump power [15]. A schematic diagram for such a system is shown in Fig. 2(a). Two types of pump lasers were used here, including a commercially available broadband ( $8 \mathrm{~nm} 3 \mathrm{~dB}$ bandwidth) fibre laser, and semiconductor laser diodes $(0.8 \mathrm{~nm} 3 \mathrm{~dB}$ bandwidth). For semiconductor laser diodes, the pump output was depolarised by combining two laser diodes through a polarisation beam combiner. The RIN levels of the two lasers were similar $(\sim-135 \mathrm{~dB} / \mathrm{Hz})$. Two power levels were tested for each type of FW-pump.
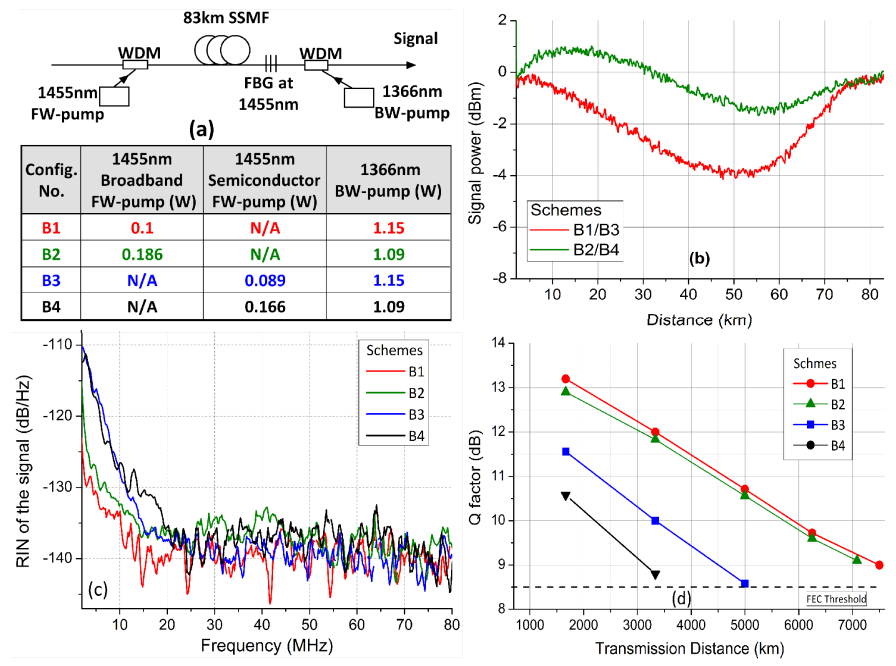

Figure 2. (a). Schematic of Raman fibre laser based amplifier using first order FW pumping. (b). Signal power profiles along the span using different FW pump power. (c). RIN of the signal using different FW pump/power. (d). Q factors versus transmission distances using all the schemes.

Signal power profiles along the fibre are shown in Fig. 2(b). Due to the same pump wavelength, signal power profiles were only related to the pump power regardless of the pump types. Similarly, the signal gain can be seen near the input section of the fibre span. In Fig. 2(c), using broadband pump, the signal RIN was lower than using narrowband semiconductor pump lasers, in particularly at low frequency range. This means the signal would be more likely to be distorted by RIN from the semiconductor pump lasers. Transmission performances are presented in Fig. 2(d). The maximum reach was up to $7499 \mathrm{~km}$ when using broadband first order pump, in comparison with $7082 \mathrm{~km}$ using BW-pumping only. However, the reach was decreased to $5000 \mathrm{~km}$ using $89 \mathrm{~mW}$ semiconductor laser and $3333 \mathrm{~km}$ using $166 \mathrm{~mW}$ semiconductor laser. Both pump lasers had similar and relatively low RIN levels, but the broader bandwidth of the fibre laser led to the suppression of the RIN penalty without sacrificing the Raman gain efficiency [16].

\section{FW-propagated dual order pumping}

Scheme A used the FW pump at 1366nm which the pump wavelength was two Stokes shift from the signal wavelength in $\mathrm{C}$ band. Due to low Raman gain efficiency, the RIN transfer was suppressed. In this scheme, we deployed a similar mechanism to reduce the RIN transfer. High power pumps at $1365 \mathrm{~nm}$ and low power seed at $1425 \mathrm{~nm}$ formed dual order FW-pumping. $1425 \mathrm{~nm}$ was chosen to generate an indirect and low Raman gain to avoid the RIN transfer. In addition, the $1425 \mathrm{~nm}$ FW pump seed could improve the noise figure in low $\mathrm{C}$ band (i.e. 1530nm) [17,18]. Scheme C used four-wavelength first order BW-pumped lasers to provide flat gain over $70 \mathrm{~nm}$ between $1530 \mathrm{~nm}$ and $1600 \mathrm{~nm}$. The schematics are shown in Fig. 3(a) and three pump power combinations were used. Full pump power list can be found in [17].
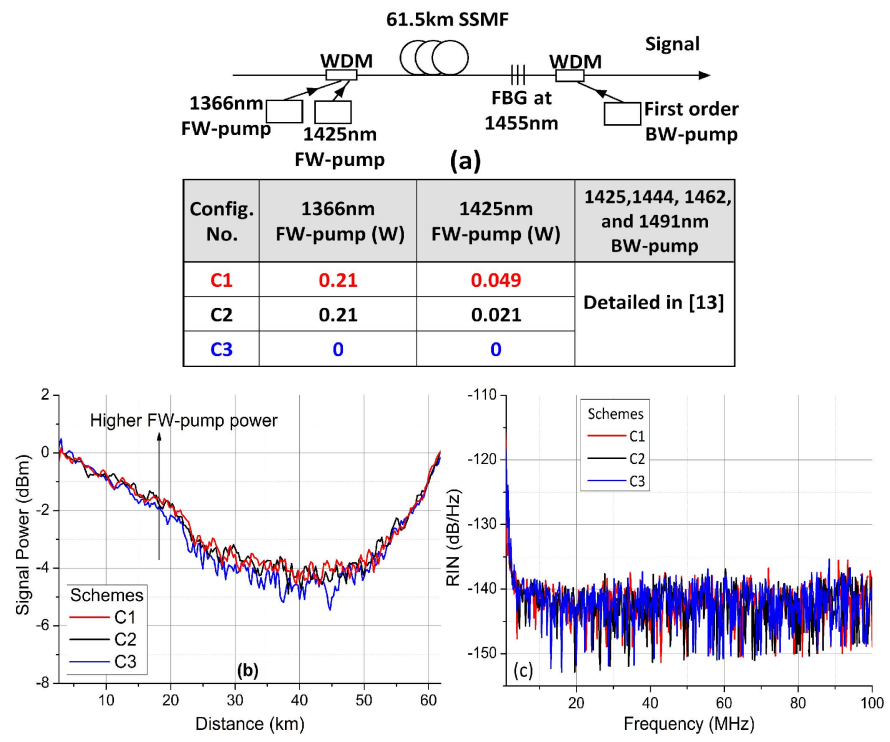

Figure 3. (a). Schematic of distributed Raman amplifier using dual order FW pumping including $1366 \mathrm{~nm}$ and $1425 \mathrm{~nm}$ pump lasers. (b). Signal power profiles along the span using different FW pump power. (c). RIN of the signal using different FW pump/power.

Figure 3(b) shows signal power profiles of scheme $\mathrm{C}$ measured at $1545.32 \mathrm{~nm}$. The signal was amplified slightly near the input section when using dual order FW-pumping, due to the indirect and low gain from $1425 \mathrm{~nm}$ to $1545 \mathrm{~nm}$. However, the RIN of the signal using dual order FW-pumping also remained the same as BW-pumping only, which indicates the RIN-penalty can be potentially mitigated. We then verified the transmission performance of this amplifier scheme, and the results showed both $\mathrm{C} 1$ and $\mathrm{C} 2$ had longer maximum reach than BW-pumping only. For other dual order FW-pumping scheme to reduce the RIN transfer, M. Morimoto proposed the scheme utilizing first order incoherent pumping light (very 
broadband) and conventional second order pump laser [19], which mitigated the signal RIN and showed $0.3 \mathrm{~dB}$ Q factor improvement in a long-haul DP-16QAM transmission system.

\section{CONCLUSION}

We have reviewed different RIN mitigation techniques in distributed Raman amplification which can effectively improve the performance in long-haul transmission system, by allowing the benefit of forward-propagated pumping, such as ASE noise reduction, symmetric signal power profile along the span (midlink OPC system), and noise figure/bandwidth improvement at the low $\mathrm{C}$ band. The paper provides the guidelines of RIN reduction and performance improvement for the design of bidirectionally pumped distributed Raman amplifiers in coherent transmission systems.

\section{ACKNOWLEDGMENT}

This work was funded by UK EPSRC Programme Grant UNLOC EP/J017582/1, FP7 ITN programme ICONE (No. 608099), and industrial support from II-IV. We thank Changle Wang, Zhongyuan Sun, and Lin Zhang for providing FBGs.

\section{REFERENCES}

[1] J. Bromage, "Raman Amplification for Fiber Communications Systems," Journal of Lightwave Technology, vol. 22, pp. 79-93, 2004.

[2] J. D. Ania-Castañón et al., "Simultaneous spatial and spectral transparency in ultralong fiber lasers," Physics Review Letters, vol. 101, 123903, 2008.

[3] W. S. Pelouch, "Raman Amplification: an Enabling Technology for High-Capacity, Long-Haul Transmission," in Optical Fiber Communication Conference, OSA Technical Digest (online) (Optical Society of America, 2015), paper. W1C.1.

[4] C. R. S. Fludger et al., "Pump to signal RIN transfer in Raman fiber amplifiers," Journal of Lightwave Technology, vol. 19, pp. 1140-1148, 2001.

[5] Y. Ohki et al., "Pump laser module for co-propagating Raman amplifier," Fukukawa Review, vol. 24, pp. 6-12, 2003.

[6] M. Tan et al., "Evaluation of 100G DP-QPSK long-haul transmission performance using second order co-pumped Raman laser based amplification," Opt. Express vol. 23, pp. 22181-22189, 2015.
[7] L. Xu et al., "Experimental Verification of Relative Phase Noise in Raman Amplified Coherent Optical Communication System," Journal of Lightwave Technology, vol. 34, pp. 3711-3716, 2016.

[8] S. Faralli et al., "Unrepeated WDM Transmission Systems Based on Advanced First-Order and Higher Order Raman-Copumping Technologies," Journal of Lightwave Technology, vol. 25, pp. 35193527, 2007.

[9] H. Bissessur, "Amplifier technologies for unrepeatered links, submarine transmissions," 2013 Optical Fiber Communication Conference and Exposition and the National Fiber Optic Engineers Conference (OFC/NFOEC) 2013, pp. 1-3.

[10] P. Rosa et al., "Unrepeatered DP-QPSK Transmission Over 352.8 km SMF Using Random DFB Fiber Laser Amplification," IEEE Photonics Technology Letters, vol. 27, pp. 1189-1192, 2015.

[11] S. B. Papernyi, V. I. Karpov, and W. R. L. Clements, "Third-order cascaded Raman amplification," in Optical Fiber Communications Conference (Optical Society of America, 2002), paper FB4.

[12] M. Tan et al., "Transmission performance improvement using random DFB laser based Raman amplification and bidirectional second-order pumping," Opt. Express, vol. 24, pp. 2215-2221, 2016.

[13] M. Tan, et al., "RIN mitigation in second- order pumped Raman fibre laser based amplification," in Asia Communications and Photonics Conference, 2015, paper AM2E.6.

[14] G. Rizzelli et al., "Impact of input FBG reflectivity and forward pump power on RIN transfer in ultralong Raman laser amplifiers," Opt. Express, vol. 24, pp. 29170-29175, 2016.

[15] M. Tan et al., "RIN-Penalty Mitigation And Transmission Performance Improvement Using Forward-Propagated Broadband First Order Raman Pump," submitted to Opto-Electronics and Communications Conference (OECC), 2017

[16] M. Tan et al., "Evaluation of Long-haul Coherent Transmission Performance Using Low RIN Forward Raman Pump," Asia Communications and Photonics Conference (ACP) 2016, paper AF3D.2.

[17] M. A. Iqbal et al., "Noise Performance Improvement of Broadband Distributed Raman Amplifier Using Dual Order Bidirectional Pumping," Asia Communications and Photonics Conference (ACP) 2016, paper AF4G.2.

[18] M. A. Iqbal et al., "Performance improvement of broadband distributed Raman amplifier using bidirectional pumping with first and dual order forward pumps," 2016 18th International Conference on Transparent Optical Networks (ICTON), Trento, 2016, pp. 1-4.

[19] M. Morimoto et al., "Co-Propagating Dual-Order Distributed Raman Amplifier Utilizing Incoherent Pumping," IEEE Photonics Technology Letters, vol. 29, no. 7, pp. 567-570, 2017. 